\title{
Two-band model for magnetism and superconductivity in nickelates
}

\author{
Lun-Hui Hu 1 a and Congjun Wu $(1)$ \\ Department of Physics, University of California, San Diego, California 92093, USA
}

(Received 7 October 2019; published 30 December 2019)

\begin{abstract}
The recently discovered superconductivity in $\mathrm{Nd}_{1-x} \mathrm{Sr}_{x} \mathrm{NiO}_{2}$ provides a new opportunity for studying strongly correlated unconventional superconductivity. The single-hole $\mathrm{Ni}^{+}\left(3 d^{9}\right)$ configuration in the parent compound $\mathrm{NdNiO}_{2}$ is similar to that of $\mathrm{Cu}^{2+}$ in cuprates. We suggest that after doping, the intraorbital spin-singlet and interorbital spin-triplet double-hole (doublon) configurations of $\mathrm{Ni}^{2+}$ are competing, and we construct a twoband Hubbard model by including both the $3 d_{x^{2}-y^{2}}$ and $3 d_{x y}$ orbitals. The effective spin-orbital superexchange model in the undoped case is a variant of the SU(4) Kugel-Khomskii model augmented by symmetry-breaking terms. Upon doping, the effective exchange interactions between spin- $\frac{1}{2}$ single holes, spin-1 (triplet) doublons, and singlet doublons are derived. Possible superconducting pairing symmetries are classified in accordance to the $D_{4 h}$ crystalline symmetry, and their connections to the superexchange interactions are analyzed.
\end{abstract}

DOI: 10.1103/PhysRevResearch.1.032046

The recent exciting discovery of the nickelate superconductivity [1] has aroused a great deal of attention in the condensed-matter community [2-12]. The infinite-layer nickelate, $\mathrm{Nd}_{0.8} \mathrm{Sr}_{0.2} \mathrm{NiO}_{2}$, is synthesized on the $\mathrm{SrTiO}_{3}$ substrate, which exhibits relatively high-transition temperatures $\left(T_{c}\right)$ around $9-15 \mathrm{~K}$. The unusual electronic configuration of $\mathrm{Ni}^{+}$ $\left(3 d^{9}\right)$ is similar to that of $\mathrm{Cu}^{2+}$ in high- $T_{c}$ cuprate superconductors. During the past three decades, the high- $T_{c}$ superconductivity remains one of the most outstanding problems in condensed-matter physics [13-17]. The parent cuprate compounds are charge-transfer insulators based on the ZaanenSawatzky-Allen scheme [18] exhibiting the antiferromagnetic (AFM) long-range order. Upon chemical doping, additional holes go to the oxygen $2 p$ orbitals, and are combined with the $3 d_{x^{2}-y^{2}}$ spins of $\mathrm{Cu}^{2+}$ cations to form the Zhang-Rice singlets [19]. The $d$-wave superconductivity arises as doping suppresses the AFM long-range order [20-22]. It has been a long-lasting question if the fascinating physics in high$T_{c}$ cuprates also exists in other strongly correlated oxides. Due to its similarity to cuprates, a great deal of efforts both experimental and theoretical have been made to investigate the nickelate-based superconductivity [23-33].

Although the nickelates exhibit a similar configuration to cuprates, their behaviors are very different. The most noticeable distinction is that no evidence of magnetic ordering is experimentally observed [24,34]. One possible reason is the large charge-transfer energy, i.e., the energy difference between a single hole lying on the nickel and oxygen sites, $\Delta_{p d} \approx 9 \mathrm{eV}$ in $\mathrm{NdNiO}_{2}$, which is much larger than $\Delta_{p d} \approx$ $3 \mathrm{eV}$ in cuprates. The superexchange energy scale is estimated as $J \sim 1 / \Delta_{p d}^{2}$ [11], which is about one order smaller than

Published by the American Physical Society under the terms of the Creative Commons Attribution 4.0 International license. Further distribution of this work must maintain attribution to the author(s) and the published article's title, journal citation, and DOI. that in cuprates [35], and thus the AFM ordering is weakened. Another possibility is the self-doping effect [4,9] to the Mott insulating state, where the forming of Kondo singlets suppresses the AFM ordering [12]. The lattice constant along the $z$ axis in $\mathrm{NbNiO}_{2}$ is only about $3.4 \AA$, much smaller than that in cuprates. This leads to the dispersion along the $z$ axis from the $\mathrm{Nd} 5 d_{z^{2}}$ orbital $[1,36]$. The $\mathrm{Nd}$-originated electron pockets are found by the LDA $+U$ calculations in previous works $[23,25,26]$ and also in recent works [3-10]. Based on Ref. [4], the electron pocket volume from $\mathrm{Nd}$ electrons is estimated smaller than $4 \%$ of the Brillouin zone, and then the self-doping effect should be weak. Due to the large chargetransfer energy in nickelates, the extra holes from self-doping also appear on $\mathrm{Ni}$ sites, hence, we construct a Ni-only model as a starting point.

Due to the large charge-transfer energy in $\mathrm{NdNiO}_{2}$, extra holes by doping are commonly believed to lie on the $\mathrm{Ni}$ sites forming the double-hole configuration of $\mathrm{Ni}^{2+}\left(3 d^{8}\right)$. This is in sharp contrast to cuprates in which the doped holes lie on oxygens. So far, most work on the nickelate superconductivity view the Ni site as orbital inactive: only the $d_{x^{2}-y^{2}}$ orbital is occupied for both the single-hole configuration of $\mathrm{Ni}^{+}\left(3 d^{9}\right)$ and the doublon configuration of $\mathrm{Ni}^{2+}\left(3 d^{8}\right)$ (Here and after, we use "doublon" for the double-hole configurations of $\mathrm{Ni}^{2+}$ following the convention in literatures.) In other words, $\mathrm{Ni}^{2+}$ is often assumed to be a spin singlet.

In this Rapid Communication, we examine the orbital property of the Ni site and its role in quantum magnetism and superconductivity in nickelates. Due to Hund's coupling and the relatively small interorbital repulsion, the triplet doublon of the $\mathrm{Ni}^{2+}$ cation is a competing configuration, in which both the $d_{x^{2}-y^{2}}$ and $d_{x y}$ orbitals are occupied. Based on the twoband Hubbard model, we study the effective superexchange processes among spin- $\frac{1}{2}$ single holes, triplet doublons, and singlet doublons. Superconductivity arises due to the Cooper pairing between doublons, which actually only carry unit charge. Both singlet and triplet Cooper pairings can take place based on different doublon configurations. We also classify 
(a)

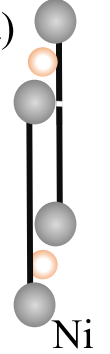

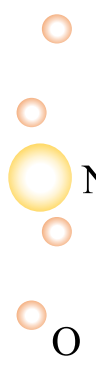

(b) one hole-doped

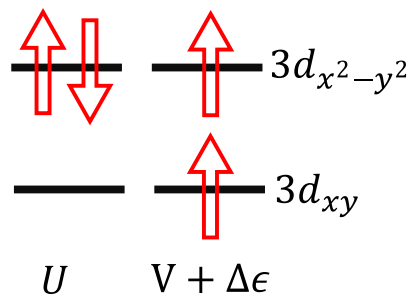

FIG. 1. (a) The crystalline structure of $\mathrm{NdNiO}_{2}$. The space-group symmetry is $P 4 / \mathrm{mmm}$. (b) The competing singlet and triplet doublon (two-hole) configurations of $\mathrm{Ni}^{2+} . U$ and $V$ are the intraorbital and interorbital interactions defined in Eq. (1). The hollow arrows represent two spin configurations of holes.

the possible pairing symmetries based on the crystalline symmetry of nickelates.

The parent compound $\mathrm{NdNiO}_{2}$ possesses the space-group crystalline symmetry of $P 4 / \mathrm{mmm}$. The Ni site is surrounded by four $\mathrm{O}^{2-}$ anions forming a planar square structure as shown in Fig. 1(a), and the $\mathrm{Ni}^{+}$cation is in the $3 d^{9}$ configuration, i.e., a single hole on the $\mathrm{Ni}$ site. The crystal-field splitting of the fivefold $3 d$ orbitals of $\mathrm{Ni}$ can be intuitively analyzed as follows based on the tetragonal symmetry: Due to the absence of the apical oxygen anions, the $d_{r^{2}-3 z^{2}}$ orbital extending along the $z$ direction has the lowest energy. It is followed by the doubly degenerate $d_{x z}$ and $d_{y z}$ orbitals, which also extend along the $z$ direction. In contrast, the energies of the in-plane orbitals are pushed higher by the negatively charged oxygen anions located in the middle of the Ni-Ni bonds: The $d_{x^{2}-y^{2}}$ orbital has the highest energy since it points to the oxygen anions, followed by the $d_{x y}$ orbital which extends along the diagonal direction of the $\mathrm{NiO}_{2}$ plane. The onsite energy difference between two highest $d_{x^{2}-y^{2}}$ and $d_{x y}$ orbitals is estimated as $\Delta \varepsilon \sim 1.38 \mathrm{eV}$ in Ref. [10]. Hence, without doping, the single hole lies in the $d_{x^{2}-y^{2}}$ orbital.

Now consider the doublon configuration when extra holes are doped. At low and intermediate levels of doping, only doublons need to be considered, and we neglect the small possibilities of three- and four-hole configurations. By keeping two highest electron orbitals, i.e., two lowest-energy orbitals of holes, two competing doublon configurations, i.e., the singlet doublon only occupying the $d_{x^{2}-y^{2}}$ orbital, and the triplet doublon occupying both $d_{x^{2}-y^{2}}$ and $d_{x y}$ orbitals, are shown in Fig. 1(b). The standard onsite two-orbital Hubbard interactions plus the onsite single-hole energy term read as

$$
\begin{aligned}
\mathcal{H}_{\text {int }}(i)= & \Delta \varepsilon n_{2}(i)+U \sum_{a=1,2} n_{a \uparrow}(i) n_{a \downarrow}(i)+V n_{1}(i) n_{2}(i) \\
& -J\left(\vec{S}_{1}(i) \cdot \vec{S}_{2}(i)-\frac{1}{4} n_{1}(i) n_{2}(i)\right)
\end{aligned}
$$

where $i$ is the site index; $a=1$ and 2 represent the $d_{x^{2}-y^{2}}$ and $d_{x y}$ orbitals, respectively; $n_{a}$ and $\vec{S}_{a}$ are the hole-number and hole-spin operators in orbital $a$, respectively; $U$ is the intraorbital interaction strength, $J$ is Hund's coupling, and $V$ is the interorbital interaction. Due to the relatively large splitting $\Delta \varepsilon$ between these two orbitals, the pairing hopping interaction is neglected. The energies of the spin-singlet and -triplet doublons are $U$ and $V+\Delta \varepsilon$, respectively. Using the estimations in Ref. [4], $U=3.8 \mathrm{eV}, V=1.9 \mathrm{eV}, J=0.7 \mathrm{eV}$ $\left(U^{\prime}=V+J=2.6 \mathrm{eV}\right.$ in their convention), the triplet energy, $V+\Delta \epsilon$, is smaller than that of the singlet. Nevertheless, accurate estimations on these parameters are very difficult at this early stage of research. Considering the uncertainty, it is reasonable to assume that their energies are close. The near degeneracy of the above competing configurations motivates us to employ the two-orbital model to describe magnetism and superconductivity in nickelates.

We define the two-band Hubbard model as $\mathcal{H}=\mathcal{H}_{t}+$ $\sum_{i} \mathcal{H}_{\text {int }}(i)$, with the hopping Hamiltonian given below as

$$
\mathcal{H}_{t}=-\sum_{\langle i j\rangle} \sum_{a=1,2} \sum_{\sigma=\uparrow, \downarrow} t_{a} \hat{c}_{a, \sigma}^{\dagger}(i) \hat{c}_{a, \sigma}(j)+\text { H.c. },
$$

where $t_{1,2}$ are the nearest-neighbor (NN) intraorbital hoppings with 1 (2) representing the $d_{x^{2}-y^{2}}$ and $d_{x y}$ orbitals, respectively. $t_{1}$ is expressed as $t_{1}=t_{1, d d}+\left(t_{1, p d}\right)^{2} / \Delta_{p d}$, where $t_{1, d d}$ is the direct overlap between $d_{x^{2}-y^{2}}$ orbitals on neighboring $\mathrm{Ni}$ sites, and the second term describes the assisted hopping via the oxygen $2 p$ orbital. Due to the large charge-transfer energy $\Delta_{p d}$ in nickelates, these two contributions are comparable. Similar analysis can be performed to $t_{2}$. The NN interorbital hoppings are forbidden due to the different symmetries of the $d_{x^{2}-y^{2}}$ and $d_{x y}$ orbitals. For simplicity, we neglect the difference between these two hopping integrals by setting $t_{1}=t_{2}=t_{0}$.

Superconducting gap-function symmetries are a central problem of unconventional superconductivity. At the current stage, this problem remains difficult for nickelate superconductors. Below we classify gap-function symmetries enriched by the multiorbital structure to provide guidance for later research. These symmetries according to the $D_{4 h}$ point-group representations [37] are $A_{1 g(u)}, A_{2 g(u)}, B_{1, g(u)}, B_{2, g(u)}$, and $E_{g(u)}$, where the subscript 1 (2) represents the even (odd) parity of the reflection with respect to the $x y$ or $y z$ planes; $g(u)$ denotes the even (odd) parity with respect to inversion; $A, B$, and $E$ indicate the discrete orbital angular momenta of 0,2 , and \pm 1 , respectively. We first consider the gap functions in the spin-singlet channel. They are represented in the two-orbital formalism as

$$
\hat{\Delta}(\mathbf{k})=\psi(\mathbf{k}) i \sigma_{y} M,
$$

where $i \sigma_{y}$ is the charge-conjugation matrix, and $M$ is the orbital pairing matrix. We use $\tau_{x, y, z}$ for the Pauli matrices in the orbital channel, where $\tau_{z}= \pm \frac{1}{2}$ refers to the $d_{x^{2}-y^{2}}$ and $d_{x y}$ orbitals, respectively, and $\tau_{0}$ is the identity matrix. The Fermi statistics imposes the constraint $\hat{\Delta}(\mathbf{k})=-\hat{\Delta}^{T}(-\mathbf{k})$. The gap functions in the spin-singlet even-parity channels are listed in Table I in which $\tau_{0, z}$ refer to the intraorbital pairing, and $\tau_{x}$ refers to the interorbital symmetric pairing. Considering the possible ferromagnetic fluctuations in nickelate superconductors, we also consider the triplet pairings

$$
\hat{\Delta}(\mathbf{k})=\mathbf{d}(\mathbf{k}) \cdot \boldsymbol{\sigma} i \sigma_{y} M,
$$

where $\mathbf{d}(\mathbf{k})$ is the so-called $d$-vector for triplet superconductors. The odd-parity triplet pairing gap functions are listed in Table II. Due to the orbital dependence, gap functions can 
TABLE I. The form factors of $\psi(\mathbf{k})$ for the even-parity, spinsinglet, and orbital-symmetric gap functions in Eq. (3).

\begin{tabular}{lcc}
\hline \hline$D_{4 h}$ & $\psi(\mathbf{k})$ & $M$ \\
\hline$A_{1 g}$ & $\cos k_{x}+\cos k_{y}, \cos k_{z}$ & $\tau_{0}, \tau_{z}$ \\
& $\sin k_{x} \sin k_{y}\left(\cos k_{x}-\cos k_{y}\right)$ & $\tau_{x}$ \\
$A_{2 g}$ & $\sin k_{x} \sin k_{y}\left(\cos k_{x}-\cos k_{y}\right)$ & $\tau_{0}, \tau_{z}$ \\
& $\cos k_{x}+\cos k_{y}, \cos k_{z}$ & $\tau_{x}$ \\
$B_{1 g}$ & $\cos k_{x}-\cos k_{y}$ & $\tau_{0}, \tau_{z}$ \\
& $\sin k_{x} \sin k_{y}$ & $\tau_{1}$ \\
$B_{2 g}$ & $\sin k_{x} \sin k_{y}$ & $\tau_{0}, \tau_{z}$ \\
& $\cos k_{x}-\cos k_{y}$ & $\tau_{x}$ \\
$E_{g}$ & $\left(\sin k_{x} \sin k_{z}, \sin k_{y} \sin k_{z}\right)$ & $\tau_{0}, \tau_{x}, \tau_{z}$ \\
\hline \hline
\end{tabular}

also be odd-parity spin singlet and even-parity spin triplet, nevertheless, they are unlikely to be relevant to the nickelate superconductivity based on the analysis later in this Rapid Communication. The hoppings of the $d_{x^{2}-y^{2}}$ and $d_{x y}$ orbitals along the $z$ direction are small, nevertheless, for completeness, we still keep the $k_{z}$-dependent gap functions.

Now, we consider the strong coupling aspect of the nickelate physics. The effective model in the strong interaction limit is constructed below via the second-order perturbation theory. The undoped configuration corresponds to the $\frac{1}{4}$-filling of holes in two bands, i.e., a spin- $\frac{1}{2}$ hole on each site. The effective superexchange model of the bond $\langle i j\rangle$ can be derived as

$$
\begin{aligned}
H_{e x}(i j)= & -\Delta \varepsilon\left(\tau_{z}(i)+\tau_{z}(j)\right)-J_{\mathrm{FM}} P_{t}^{s}(i j) P_{s}^{o}(i j) \\
& -J_{\mathrm{AF}} P_{s}^{s}(i j)\left(\tau_{z}(i)+\frac{1}{2}\right)\left(\tau_{z}(j)+\frac{1}{2}\right),
\end{aligned}
$$

where $P_{t}^{s}=\vec{S}(i) \cdot \vec{S}(j)+\frac{3}{4} n(i) n(j)$ and $P_{s}^{s}=-\vec{S}(i) \cdot \vec{S}(j)+$ $\frac{1}{4} n(i) n(j)$ are the projection operators to the bond spin-triplet and -singlet sectors, respectively, with $n(i)$ the hole number on site $i ; P_{s}^{o}=-\vec{\tau}_{i} \cdot \vec{\tau}_{j}+\frac{1}{4} n(i) n(j)$ is the projection operator in the orbital singlet channel. The orbital-flipping superexchange process is ferromagnetic (FM) represented by $J_{\mathrm{FM}} \approx 4 t_{0}^{2} / V$, while the spin-flipping superexchange process is represented by $J_{\mathrm{AF}} \approx 4 t_{0}^{2} / U$ is AFM. Due to the onsite energy splitting, only the configuration with both holes in the $d_{x^{2}-y^{2}}$ orbital is taken into account in the AFM superexchange. Equation (5) is a variant of the SU(4) Kugel-Khomskii spin-orbital model augmented by symmetry breakings [38,39]. Consider an AFM ordered Néel configuration with all holes lying in the $d_{x^{2}-y^{2}}$

TABLE II. The $d$-vectors for the spin triplet, odd-parity, and orbital-symmetric pairing gap functions in Eq. (4).

\begin{tabular}{lcc}
\hline \hline$D_{4 h}$ & $\mathbf{d}(\mathbf{k})$ & $M$ \\
\hline$A_{1 u}$ & $\sin k_{z} \hat{z}, \sin k_{x} \hat{y}+\sin k_{y} \hat{x}$ & $\tau_{0}, \tau_{z}$ \\
& $\sin k_{y} \hat{y}-\sin k_{x} \hat{x}$ & $\tau_{x}$ \\
$A_{2 u}$ & $\sin k_{y} \hat{y}-\sin k_{x} \hat{x}$ & $\tau_{0}, \tau_{z}$ \\
& $\sin k_{z} \hat{z}, \sin k_{x} \hat{y}+\sin k_{y} \hat{x}$ & $\tau_{x}$ \\
$B_{1 u}$ & $\sin k_{x} \hat{y}-\sin k_{y} \hat{x}$ & $\tau_{0}, \tau_{z}$ \\
& $\sin k_{y} \hat{y}+\sin k_{x} \hat{x}$ & $\tau_{x}$ \\
$B_{2 u}$ & $\sin k_{y} \hat{y}+\sin k_{x} \hat{x}$ & $\tau_{0}, \tau_{z}$ \\
& $\sin k_{x} \hat{y}-\sin k_{y} \hat{x}$ & $\tau_{x}$ \\
$E_{u}$ & $\left(\sin k_{x}, \sin k_{y}\right) \hat{z}, \sin k_{z}(\hat{x}, \hat{y})$ & $\tau_{0}, \tau_{x}, \tau_{z}$ \\
\hline \hline
\end{tabular}
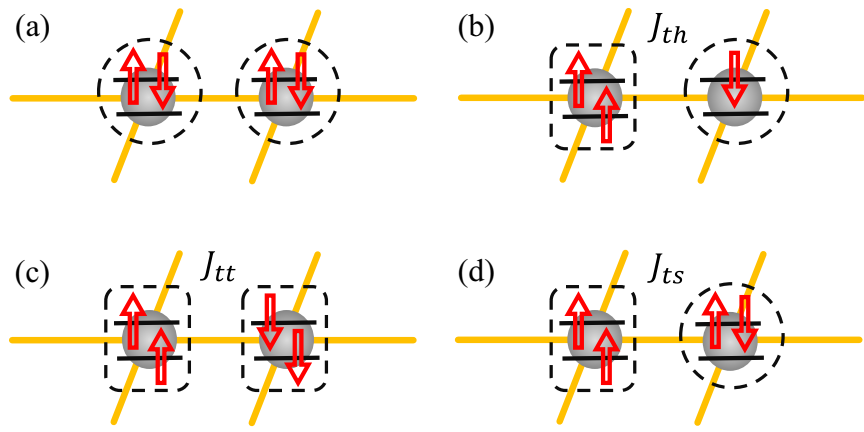

FIG. 2. Bond configurations with doublons and single holes. (a) Two neighboring singlet doublons. (b) A triplet doublon and a spin- $\frac{1}{2}$ single hole. (c) Two neighboring triplet doublons. (d) A triplet doublon and a singlet doublon.

orbital. Let us flip a hole's spin and put it into the $d_{x y}$ orbital. The exchange energy gain is roughly $\Delta E_{e x}=2 z t_{0}^{2}\left(\frac{1}{V}-\frac{1}{U}\right)$ with $z=4$ the coordination number. It is difficult to precisely estimate $\Delta E_{e x}$. It should be significantly smaller than $\Delta \varepsilon$, nevertheless, conceivably, they are still at the same order. Hence, the AFM ordering tendency would be significantly reduced, which is in agreement with the absence of AFM long-range order in experiments. Nevertheless, in the undoped case, hole remains in the $d_{x^{2}-y^{2}}$ orbital as described by

$$
H_{e x}(i j)=\tilde{J}_{\mathrm{AF}}\left(\vec{S}(i) \cdot \vec{S}(j)-\frac{1}{4} n_{i} n_{j}\right),
$$

where $\tilde{J}_{\mathrm{AF}}$ represents the reduced AFM exchange by orbital fluctuations.

Next, we construct the low-energy superexchange Hamiltonians after doping, which include both doublons and single holes, via the second-order perturbation theory. The possibility of a single hole in the $d_{x y}$ orbital is neglected for the onsite energy splitting. There is no exchange interaction between two neighboring singlet doublons [Fig. 2(a)], and no exchange interaction between a singlet doublon and a single spin- $\frac{1}{2}$ hole, either. In contrast, between a triplet doublon and a neighboring single spin- $\frac{1}{2}$ hole shown in Fig. 2(b), the superexchange interaction is ferrimagnetic,

$$
H_{e x}^{t h}(i j)=J_{t h}\left(\vec{T}_{i} \cdot \vec{S}_{j}-\frac{1}{4} n_{i} n_{j}\right),
$$

where $\vec{T}$ is the spin-1 operator of the triplet doublon, and $J_{t h}=$ $\frac{3 t^{2}}{2}\left(\frac{1}{U-V}+\frac{1}{U+V+J / 2}\right)$. Furthermore, the superexchange interaction also exists between two neighboring triplet doublons [Fig. 2(c)], which is described by the spin-1 AFM Heisenberg model as

$$
H_{e x}^{t t}(i j)=J_{t t}\left(\vec{T}_{i} \cdot \vec{T}_{j}-\frac{1}{4} n_{i} n_{j}\right),
$$

with $J_{t t}=\frac{2 t^{2}}{U+J / 2}$. Finally, if we bring a triplet and a singlet doublon together [Fig. 2(d)], their exchange interaction is described by

$$
\begin{aligned}
H_{e x}^{t s}(i j)= & -J_{t s}\left(d_{m}^{\dagger}(i) d_{0}(i) d_{0}^{\dagger}(j) d_{m}(j)+\text { H.c. }\right) \\
& +J_{t s}\left(d_{m}^{\dagger}(i) d_{m}(i) d_{0}^{\dagger}(j) d_{0}(j)\right. \\
& \left.+d_{0}^{\dagger}(i) d_{0}(i) d_{m}^{\dagger}(j) d_{m}(j)\right),
\end{aligned}
$$

where $d_{0, \pm 1}^{\dagger}$ are creation operators for doublons and $J_{t s}=$ $\frac{4 t^{2}}{V+J / 2}$. In addition to superexchange interactions, a doublon 
and a single hole can switch their positions. For the singlet doublon and a single hole, it is simply a straightforward hopping process. The switching between a triplet doublon and a single hole is described by

$$
\begin{aligned}
H_{t}^{t h}(i j)= & -t^{\prime} \sum_{m \sigma ; m^{\prime} \sigma^{\prime}}\left\{\left\langle j j_{z} \mid 1 m \frac{1}{2} \sigma\right\rangle\left\langle j j_{z} \mid 1 m^{\prime} \frac{1}{2} \sigma^{\prime}\right\rangle\right. \\
& \left.\times d_{m}^{\dagger}(i) c_{1 \sigma}^{\dagger}(j) c_{1 \sigma^{\prime}}(i) d_{m^{\prime}}(j)+\text { H.c. }\right\},
\end{aligned}
$$

where $\langle\ldots \mid \ldots\rangle$ are the Clebsch-Gordan coefficients between spin- 1 and spin- $\frac{1}{2}$ sectors, and $t^{\prime}$ is at the same order of $t_{0}$.

Now consider the glue for superconductivity based on the above superexchange picture. Compared to the undoped case, the doublon charge is one instead of two compared to the background of single holes. Pairing of two doublons leads to superconductivity. An effective attraction between two singlet doublons at neighboring sites is at the energy scale of $\Delta E_{s s}=-\frac{1}{2} \tilde{J}_{\mathrm{AF}}$. Bringing two neighboring triplet doublons together, the energy difference compared to when they are apart is $\Delta E_{t t}=2 J_{t h}-2 J_{t t}-\frac{1}{2} \tilde{J}_{\mathrm{AF}}$. Finally, bringing a triplet and a singlet doublon together, the energy difference is $\Delta E_{t s}=J_{t h}-J_{t s}-\frac{1}{2} \tilde{J}_{\mathrm{AF}}$. When each of the above quantities becomes negative, it means an effective attraction in the corresponding channel. Both cases of two singlet doublons and two triplet doublons can form spin-singlet Cooper pairing, and the pairing of a singlet doublon and a triplet doublon gives rise to a triplet pairing superconductivity. Due to the relatively large value of $J_{t h}$, the pairing strength of triplet doublons is weak if not completely suppressed.

Next, we connect the above doublon pairing picture to the previous analysis on gap-function symmetries. Consider a Ni-Ni bond $\langle i j\rangle$. In the absence of doping, we take the bond singlet state with one hole in the $d_{x^{2}-y^{2}}$ orbital on each site as the background state $\left|\Psi_{0}\right\rangle$. Furthermore, the state of two singlet doublons [Fig. 2(a)] is denoted as $\left|\Psi_{s s}\right\rangle$, and that of two triplet doublons [Fig. 2(c)] is denoted as $\left|\Psi_{d d}\right\rangle$. These states can be connected to $\left|\Psi_{0}\right\rangle$ via the pairing operators $\chi_{i j}^{\dagger, s s(d d)}=$ $\frac{1}{\sqrt{2}}\left[\hat{c}_{a \uparrow}^{\dagger}(i) \hat{c}_{a \downarrow}^{\dagger}(j)-\hat{c}_{a \downarrow}^{\dagger}(i) \hat{c}_{a \uparrow}^{\dagger}(j)\right]$ with $a=d_{x^{2}-y^{2}}$ for $\chi_{i j}^{\dagger, s s}$ and $d_{x y}$ for $\chi_{i j}^{\dagger, d d}$, such that $\left\langle\Psi_{s s(d d)}\left|\chi_{i j}^{\dagger, s s(d d)}\right| \Psi_{0}\right\rangle \neq 0$. Then the orbital pairing matrices for $\chi_{i j}^{\dagger, s s(d d)}$ correspond to $\tau_{0} \mp \tau_{z}$, respectively. According to Table I, the plausible pairing symmetries are $A_{1 g}$ ( $s$ wave), and $B_{1 g}\left(d_{x^{2}-y^{2}}\right.$ wave), with the form factors $\cos k_{x} \pm \cos k_{y}$, respectively. As for the triplet pairing between singlet and triplet doublons, the bonding state corresponds to the odd-parity combinations of the configuration in Fig. 2(d) and its parity partner. The corresponding pairing operators are orbital symmetric and odd parity: $A_{1 u}$, $A_{2 u}, B_{1 u}, B_{2 u}, E_{u}$. Their $d$-vector configurations exhibit the $p$-wave orbital symmetries of $\sin k_{x}$ and $\sin k_{y}$ and are shown in Table II.

Energetically, it is more favorable if the gap function nodes are away from the van Hove singularities of density of states. Current band structure calculations show large density of states around $(0, \pi)$ and $(\pi, 0)$. Hence, the $B_{1 g}\left(d_{x^{2}-y^{2}}\right)$ singlet pairing symmetry is probably the dominant one. The singlet $A_{1 g}$ and $p$-wave triplet pairings are competing pairing symmetries but less favorable. Since the singlet doublon pairing

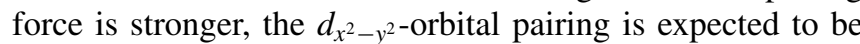
more dominant than that in the $d_{x y}$ orbital.

We note that a similar two-band model based on the nearly degenerate $e_{g}$ orbitals of $d_{x^{2}-y^{2}}$ and $d_{r^{2}-3 z^{2}}$ was recently developed for $\mathrm{CuO}_{2}$ monolayers [40], as well as the high- $T_{C}$ cuprate superconductor $\mathrm{Ba}_{2} \mathrm{CuO}_{3+x}$ [41]. In nickelates the planar $B_{2 g}$ orbital $d_{x y}$ replaces the $z$-directional $d_{r^{2}-3 z^{2}}$ orbital in the $\mathrm{CuO}_{2}$ plane whose symmetry is reduced to $A_{1 g}$ under the tetragonal symmetry. Due to the different orbital symmetries, the interorbital pairing symmetries are different in these two classes of systems.

Conclusion. We suggest that the low-energy physics in $\mathrm{Nd}_{1-x} \mathrm{Sr}_{x} \mathrm{NiO}_{2}$ is captured by the two-orbital model. The second-order perturbation theory is employed to derive the effective Hamiltonians in both cases with and without dopings. In the absence of doping, it is a variant of the SU(4) Kugel-Khomskii spin-orbital model subject to symmetrybreaking terms. It shows the competition between FM and AFM exchanges, thus, the magnetic tendency is significantly suppressed. When additional holes are doped into nickelates, two competing configurations of $\mathrm{Ni}^{2+}$ appear: the intraorbital singlet doublon and the interorbital triplet doublon. The superexchange interactions among two types of doublons and single holes are derived, and doublon pairings are studied in the superexchange picture. Possible pairing symmetries are analyzed based on the $D_{4 h}$ point group.

Note added. Recently, we became aware of Refs. [42,43], in which the triplet doublons of the $\mathrm{Ni}^{2+}$ cations are also proposed.

Acknowledgments. C.W. thanks J. Hirsch for helpful discussions. L.-H.H. and C.W. are supported by AFOSR Grant No. FA9550-14-1-0168.
[1] D. Li, K. Lee, B. Y. Wang, M. Osada, S. Crossley, H. R. Lee, Y. Cui, Y. Hikita, and H. Y. Hwang, Nature (London) 572, 624 (2019).

[2] J. E. Hirsch and F. Marsiglio, Physica C 566, 1353534 (2019).

[3] A. S. Botana and M. R. Norman, arXiv:1908.10946.

[4] H. Sakakibara, H. Usui, K. Suzuki, T. Kotani, H. Aoki, and K. Kuroki, arXiv: 1909.00060.

[5] M. Hepting, D. Li, C. Jia, H. Lu, E. Paris, Y. Tseng, X. Feng, M. Osada, E. Been, Y. Hikita et al., arXiv:1909.02678.
[6] Y. Nomura, M. Hirayama, T. Tadano, Y. Yoshimoto, K. Nakamura, and R. Arita, Phys. Rev. B 100, 205138 (2019).

[7] J. Gao, Z. Wang, C. Fang, and H. Weng, arXiv:1909.04657.

[8] S. Ryee, H. Yoon, T. J. Kim, M. Y. Jeong, and M. J. Han, arXiv:1909.05824.

[9] H. Zhang, L. Jin, S. Wang, B. Xi, X. Shi, F. Ye, and J.-W. Mei, arXiv: 1909.07427.

[10] X. Wu, D. Di Sante, T. Schwemmer, W. Hanke, H. Y. Hwang, S. Raghu, and R. Thomale, arXiv:1909.03015. 
[11] M. Jiang, M. Berciu, and G. A. Sawatzky, arXiv:1909.02557.

[12] G.-M. Zhang, Y.-F. Yang, and F.-C. Zhang, arXiv:1909.11845.

[13] J. G. Bednorz and K. A. Müller, Z. Phys. B 64, 189 (1986).

[14] J. G. Bednorz and K. A. Müller, Rev. Mod. Phys. 60, 585 (1988).

[15] P. W. Anderson, Science 235, 1196 (1987).

[16] P. W. Anderson, P. Lee, M. Randeria, T. Rice, N. Trivedi, and F. Zhang, J. Phys.: Condens. Matter 16, R755 (2004).

[17] P. A. Lee, N. Nagaosa, and X.-G. Wen, Rev. Mod. Phys. 78, 17 (2006).

[18] J. Zaanen, G. A. Sawatzky, and J. W. Allen, Phys. Rev. Lett. 55, 418 (1985).

[19] F. C. Zhang and T. M. Rice, Phys. Rev. B 37, 3759 (1988).

[20] Z. X. Shen, D. S. Dessau, B. O. Wells, D. M. King, W. E. Spicer, A. J. Arko, D. Marshall, L. W. Lombardo, A. Kapitulnik, P. Dickinson, S. Doniach, J. Di Carlo, A. G. Loeser, and C. H. Park, Phys. Rev. Lett. 70, 1553 (1993).

[21] D. A. Wollman, D. J. Van Harlingen, W. C. Lee, D. M. Ginsberg, and A. J. Leggett, Phys. Rev. Lett. 71, 2134 (1993).

[22] C. C. Tsuei, J. R. Kirtley, C. C. Chi, Lock See Yu-Jahnes, A. Gupta, T. Shaw, J. Z. Sun, and M. B. Ketchen, Phys. Rev. Lett. 73, 593 (1994).

[23] V. I. Anisimov, D. Bukhvalov, and T. M. Rice, Phys. Rev. B 59, 7901 (1999).

[24] M. Hayward, M. Green, M. Rosseinsky, and J. Sloan, J. Am. Chem. Soc. 121, 8843 (1999).

[25] K.-W. Lee and W. E. Pickett, Phys. Rev. B 70, 165109 (2004).

[26] J. Chaloupka and G. Khaliullin, Phys. Rev. Lett. 100, 016404 (2008).

[27] P. Hansmann, X. Yang, A. Toschi, G. Khaliullin, O. K. Andersen, and K. Held, Phys. Rev. Lett. 103, 016401 (2009).
[28] A. Boris, Y. Matiks, E. Benckiser, A. Frano, P. Popovich, V. Hinkov, P. Wochner, M. Castro-Colin, E. Detemple, V. K. Malik et al., Science 332, 937 (2011).

[29] E. Benckiser, M. W. Haverkort, S. Brück, E. Goering, S. Macke, A. Frañó, X. Yang, O. K. Andersen, G. Cristiani, H.-U. Habermeier et al., Nat. Mater. 10, 189 (2011).

[30] A. S. Disa, D. P. Kumah, A. Malashevich, H. Chen, D. A. Arena, E. D. Specht, S. Ismail-Beigi, F. J. Walker, and C. H. Ahn, Phys. Rev. Lett. 114, 026801 (2015).

[31] S. Middey, J. Chakhalian, P. Mahadevan, J. Freeland, A. J. Millis, and D. Sarma, Annu. Rev. Mater. Res. 46, 305 (2016).

[32] A. S. Botana, V. Pardo, and M. R. Norman, Phys. Rev. Mater. 1, 021801 (2017).

[33] J. Zhang, A. Botana, J. Freeland, D. Phelan, H. Zheng, V. Pardo, M. Norman, and J. Mitchell, Nat. Phys. 13, 864 (2017).

[34] M. Hayward and M. Rosseinsky, Solid State Sci. 5, 839 (2003).

[35] J. Zaanen and G. A. Sawatzky, Can. J. Phys. 65, 1262 (1987).

[36] A. Ikeda, Y. Krockenberger, H. Irie, M. Naito, and $H$. Yamamoto, Appl. Phys. Express 9, 061101 (2016).

[37] M. Sigrist and K. Ueda, Rev. Mod. Phys. 63, 239 (1991).

[38] K. I. Kugel and D. I. Khomskii, Sov. Phys. Usp. 25, 231 (1982).

[39] Y.-Q. Li, M. Ma, D. N. Shi, and F. C. Zhang, Phys. Rev. Lett. 81, 3527 (1998).

[40] K. Jiang, X. Wu, J. Hu, and Z. Wang, Phys. Rev. Lett. 121, 227002 (2018).

[41] C. Le, K. Jiang, Y. Li, S. Qin, Z. Wang, F. Zhang, and J. Hu, arXiv: 1909.12620.

[42] Y.-H. Zhang and A. Vishwanath, arXiv:1909.12865.

[43] P. Werner and S. Hoshino, arXiv:1910.00473. 